\title{
Synthesis and characterization of nanosized calcium phosphates by flame spray pyrolysis, and their effect on osteogenic differentiation of stem cells
}

\author{
Sibel Ataol • Ayşen Tezcaner • Ozgur Duygulu • \\ Dilek Keskin • Nesrin E. Machin
}

Received: 8 August 2014/Accepted: 2 February 2015/Published online: 14 February 2015

(C) Springer Science+Business Media Dordrecht 2015

\begin{abstract}
The present study evaluates the synthesis of biocompatible osteoconductive and osteoinductive nano calcium phosphate $(\mathrm{CaP})$ particles by industrially applied, aerosol-derived flame spray pyrolysis method for biomedical field. Calcium phosphate nanoparticles were produced in a range of calciumto-phosphorus ratio, (1.20-2.19) in order to analyze the morphology and crystallinity changes, and to test the bioactivity of particles. The characterization results confirmed that nanometer-sized, spherical calcium phosphate particles were produced. The
\end{abstract}

Guest Editors: Mustafa Culha, Rawil F. Fakhrullin, Ratnesh Lal

This article is part of the topical collection on

Nanobiotechnology

Electronic supplementary material The online version of this article (doi:10.1007/s11051-015-2901-0) contains supplementary material, which is available to authorized users.

S. Ataol · A. Tezcaner - D. Keskin

Department of Biomedical Engineering, Middle East

Technical University, 06800 Ankara, Turkey

\section{A. Tezcaner · D. Keskin}

Department of Engineering Sciences, Middle East

Technical University, 06800 Ankara, Turkey

\section{A. Tezcaner $\cdot$ D. Keskin}

BIOMATEN Center of Excellence in Biomaterials and Tissue Engineering, Middle East Technical University, 06800 Ankara, Turkey average primary particle size was determined as $23 \mathrm{~nm}$ by counting more than 500 particles in TEM pictures. XRD patterns, HRTEM, SAED, and SEM analyses revealed the amorphous nature of the asprepared nano calcium phosphate particles at low $\mathrm{Ca} / \mathrm{P}$ ratios. Increases in the specific surface area and crystallinity were observed with the increasing $\mathrm{Ca} / \mathrm{P}$ ratio. TGA-DTA analysis showed that the thermally stable crystal phases formed after $700{ }^{\circ} \mathrm{C}$. Cell culture studies were conducted with urine-derived stem cells that possess the characteristics of mesenchymal stem cells. Synthesized amorphous nanoparticles did not have cytotoxic effect at $5-50 \mu \mathrm{g} / \mathrm{ml}$ concentration range. Cells treated with the as-prepared nanoparticles had higher alkaline phosphatase (ALP) enzyme activity than control cells, indicating osteogenic differentiation of cells. A slight decrease in ALP activity of cells treated with two highest $\mathrm{Ca}: \mathrm{P}$ ratios at $50 \mu \mathrm{g} / \mathrm{ml}$ concentration was observed at day 7 . The findings suggest that calcium phosphate nanoparticles

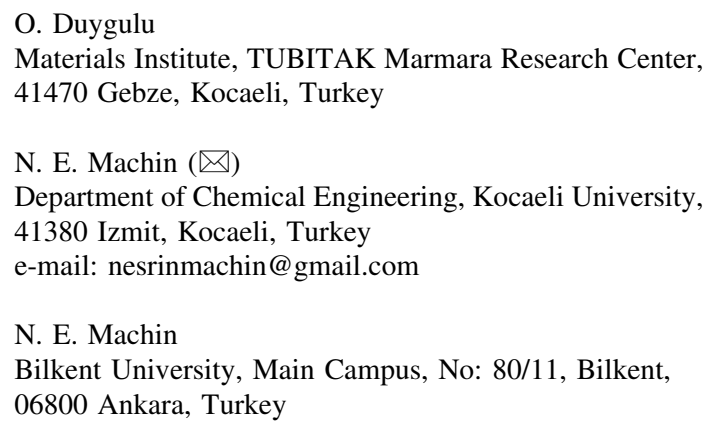


produced in this work have a potential to be used as biomaterials in biomedical applications.

Keywords Flame spray pyrolysis - Nanoparticles · Calcium phosphates · USC · Osteogenic differentiation $\cdot$ Nanobiotechnology $\cdot$ Nanomedicine

\section{Introduction}

Every year, the worldwide records of bone disorders and bone defects due to trauma, illnesses, or poor physical activities increase steeply. In medical treatment of such disorders, damaged bone tissue is replaced with metallic implants or filled with supportive active materials depending on the scar tissue size. In both treatment approaches, the critical point is the presence of active calcium phosphate-derived materials whether on the surface of metal implants or in filler bone cement. These active materials are chosen and designed to support osteointegration of the implant. They are designed to trigger cellular regeneration by bonding biomaterial to host bone tissue. These biological phenomena could be achieved only by mimicking the natural bone microenvironment for cells. Depending on bone tissue type and location, the calcium phosphate ceramic content and the properties of tissue differ. In general, the calcium phosphate phase of bone consists of $65-70 \%$ of bone tissue (Sadat-Shojai et al. 2013). In natural bone and similar calcified tissues, biominerals have two distinct features: they are nanostructured, and they have low crystallinity (Alves Cardoso et al. 2012; Sadat-Shojai et al. 2013). In biological systems, natural biominerals are favorable to be nanostructured; thus, they can disperse through organism by mobility and dissolubility kinetics of nanomaterials. As the particle gets smaller in size, the mobility in body will be higher. Large surface-to-volume ratio of the nanosized particles provides high crystal growth potential and thereby biological mineralization. Since large surface area increases the surface ionic interaction of calcium phosphate particles with biological environment, $\mathrm{Ca}^{+2}$ ions react with free ions, and crystal apatite nucleation is favoured, resulting in higher biomineralization (Barrère et al. 2006; Dorozhkin 2010; Blokhuis and Arts 2011; Chen et al. 2012). However, the increasing concentration of smaller nanoparticles results in large and immobile aggregates, which limits the exposure dose for nanoparticles to parts per million for biomedical and toxicology studies (Stark 2011).

Synthetic calcium phosphates are widely used in biomedical field as a bone substitute and bone-filling cements due to their chemical similarity to calcium phosphates in bone. The requirements for calcium phosphates to be considered as biomaterials are biocompatibility, biodegradability, bioactivity, osteoconductivity, and osteoinductivity. The biocompatibility is a terminology which is used to define the compatibility of materials with living body both at systemic and cellular levels. The biodegradability means the degradation of material in body in time, which results from enzymatic cleavage, or ambient conditions like $\mathrm{pH}$ changes, temperature changes, etc. Bioactive materials are materials that form an organic bond with the host tissue. Calcium phosphate particles are osteoconductive and osteoinductive materials (Cho et al. 2010; Lobo and Livingston Arinzeh 2010). Osteoconductivity and osteoinductivity are considered as an indicator of bioactivity. Osteoconductivity is used to define the ability of material in providing a structure to which cells can adhere, proliferate, and further propagate for bone tissue formation. Osteoinductivity defines the potential of materials for triggering osteogenic differentiation (Blokhuis and Arts 2011; Salinas et al. 2013). In this study, calcium phosphate particles were evaluated for their biocompatibility and bioactivity properties.

Calcium phosphates are synthesized using various methods that are classified as high-temperature process, wet- and dry-based methods (Loher et al. 2005; Teoh et al. 2010; Sadat-Shojai et al. 2013). Wet-based methods are chemical precipitation, sol-gel method, and hydrolysis method. Dry-based methods are solidstate method and mechano-chemical synthesis. Among these three methods, wet-based chemical precipitation is widely preferred, since it is easy to apply and incurs low cost. In this synthesis method, precursors are dissolved in aqueous medium at low temperatures. Organic solvent is not used; therefore, the method is cost effective and environmentally friendly (Mohn et al. 2011). The precursors react at ambient temperature and pressure by controlling $\mathrm{pH}$ of the mixture continuously. Wet-based synthesis method is stated as an advantageous method to control the morphology and the mean size of the produced calcium phosphate powders by tuning the parameters 
externally (Sadat-Shojai et al. 2013). However, the low temperature affects the reaction efficiency and leads to impurity of different phases of calcium phosphates. Meanwhile, the reaction results with low crystalline particles (Cho et al. 2009). Dry-based solid-state synthesis method involves mixing of precursor's powder. The precursors are mixed; milled, and sintered at elevated temperatures, and a thermally stable product is obtained (Tofighi and Palazzolo 2005). Since the reaction takes place in a dry environment, ions do not interact in solid as in solvent, resulting in a lower yield than wet-based methods (Sadat-Shojai et al. 2013). The particles can be synthesized in nanosizes by dry methods. When they are used for biomedical applications, they show reduced contact with body fluid, hindering resorption (Zhou and Lee 2011).

Amorphous calcium phosphates (ACPs) are one of the widely used biomaterials for tissue engineering applications as ionic cement, coating of prosthesis, and mineral organic composites to benefit from their physicochemical behavior (Brunner et al. 2007a; Liu et al. 2008; Combes and Rey 2010; He et al. 2013). Reactivity of calcium phosphates (dissolution from the surface of material and re-precipitation as apatite) in biological environments or stimulated body fluids is called bioactivity of these biomaterials. ACPs have higher solubility in aqueous biological environment compared to crystalline ones resulting in high ionic concentration in the vicinity, initiating a recrystallization of apatite-like deposits. The physiological $\mathrm{pH}$ induces this crystallization (Somrani et al. 2003; Combes and Rey 2010). This biomineralization is initiated by the electrostatic interactions between the calcium and phosphate ions in the body fluids and the surface functional groups of the biomaterial. In amorphous materials, lower reaction energy is required for such interaction of surface functional groups and local $\mathrm{Ca}^{2+}$ and $\mathrm{PO}_{4}{ }^{3-}$ ions. This ionexchange phenomenon of resorbable calcium phosphates makes this biomaterial very attractive and preferable for their use as coating materials on implants ensuring their cohesion with host with the newly formed mineralized interface, and in composite carriers for hard tissue engineering applications ensuring improvement of the mechanical properties and the biological response by mimicking the bone matrix with biomineralization.
During bone metabolism, osteoclasts degrade the mineralized matrix of the defected site in bone, and preosteoblasts migrate to this site and bone formation proceeds. In this dynamic mechanism, free $\mathrm{Ca}^{2+}$ and $\mathrm{PO}_{4}{ }^{3-}$ ions have crucial role in cellular activities. Free ions trigger the chemotaxis of preosteoblasts to defect site, diffuse into cells, and induce expressions of differentiation and proliferation growth factors. However, the exact mechanism is still unknown (Chai et al. 2012). Higher resorbability of ACP assists natural process; thus, ACPs are osteoconductive by triggering chemotaxis and osteoinductive by inducing growth factor expression (Nagano et al. 1996; Chai et al. 2012). Since osseointegration is required in hard tissue engineering applications for longevity of implants, ACPs ensure the bone binding by dissolving rapidly, diffusing into cells, and reacting with collagen protein (Combes and Rey 2010; He et al. 2013). In aqueous environment, by high ionic interaction, ACPs show apatite-like recrystallization. The physiological $\mathrm{pH}$ induces this crystallization (Somrani et al. 2003; Combes and Rey 2010). Crystalline calcium phosphate biomaterials show higher thermal stability than natural and amorphous ones. Their dissolution rate is lower, and osseointegration process occurs in slow manner. In previous studies, implant surface coatings were studied with amorphous and highly crystalline calcium phosphates, and their properties were compared on 16 weeks' polyethersulfone (PES) plate implants. The mechanical load capacity and bone implant binding were found higher in amorphous calcium phosphate coated implants. It was stated that the high degradability of ACP facilitated interaction of implant with surrounding bone tissue. The osseointegration of implant increased the mechanical strength of implant; meanwhile, highly crystalline hydroxyapatite-loaded implants were fragile, and their bonding was weak (Nagano et al. 1996). Moreover, amorphous calcium phosphate and polymer-based composite scaffolds have been developed for bone tissue engineering applications, and in vitro biomineralization of these matrices have been conducted (Loher et al. 2006; Hild et al. 2011). The bioactivity differences of amorphous and crystalline calcium phosphates were examined. Their osteogenesis comparison was conducted with mouse osteoblasts on titanium implant surfaces. The ACPSs showed better bioactivity than crystal calcium phosphates (Brunner 
et al. 2007b; He et al. 2013). According to the study, for the higher surface area, smaller particle-sized amorphous materials showed better osteogenic differentiation on seeded cells (Somrani et al. 2003; Brunner et al. 2007b).

The improvements in nanotechnology have increased the interest in synthesis of bioceramics in nanosizes. Flame spray pyrolysis (FSP) method is a highly promising and versatile method, which enables the desired nanoparticle design and fabrication (Madler et al. 2002; Teoh et al. 2010). The precursor selection and reactor system engineering make FSP system practically feasible to produce particles with the desired properties. Previously, a variety of ceramic powders have been made by FSP system (Pratsinis 1998). The system has been also studied for synthesizing calcium phosphate bioceramics for biomedical applications (Loher et al. 2005; Maciejewski et al. 2008; Cho and Kang 2008; Dorozhkin 2010; Boccaccini et al. 2010; Zhou and Lee 2011; Hild et al. 2012; Sadat-Shojai et al. 2013).

The FSP system is scalable on particle formation, morphology, and size compared with wet and drybased synthesis methods. The choice of solvents or liquid precursors affects the combustion reaction. This provides direct control on primary particle size and phase of materials. The local temperature and residence time of particles in flame determines the crystallinity and nanoscale feature of materials (Cho and Kang 2008; Teoh et al. 2010). However, in conventional methods, to scale the particle size, milling step is required, and for phase transition, gradual calcination and sintering processes are required to be performed (Jillavenkatesa and Condreta 1998; Tofighi and Palazzolo 2005; El Briak-Benabdeslam et al. 2008; Combes and Rey 2010; Chen et al. 2012). Moreover, FSP system is a time-effective synthesis method. Conventional systems such as solgel synthesis and precipitation synthesis methods take nearly $12-15 \mathrm{~h}$ to perform, and several other drying steps and heat-treatment cycles are required (Jillavenkatesa and Condreta 1998; Fathi et al. 2008). The features of the FSP system provide advantages over the conventional production methods. Moreover, the thermal quenching in FSP reactor system while collecting particles results in thermally metastable particles (Zhou and Lee 2011). A metastable form of calcium phosphate particle synthesis may provide an opportunity in biomedical studies. In the natural biomineralization processes, the bone minerals attach on collagen fibers and provide a site for further nucleation. Ion movement and localization induces osteoblast (bone-forming cells) for regeneration (Hench and Polak 2002). In previous studies, this bone growth-inducing property was mimicked by coating carriers with amorphous metastable form of calcium phosphate particles. The histological studies showed that amorphous form has high resorbability and bioactivity because of the higher bonding affinity (Overgaard et al. 1999; Sarkar et al. 2001). In light of these advantages, in this study, we aimed to synthesize amorphous calcium phosphate nanoparticles by the FSP method with calcium acetate hydrate as a $\mathrm{Ca}$ source and propionic acid as the solvent. In addition, the effects of synthesized nanoparticles on the viability and osteogenic differentiation of the human urine-derived stem cells (USC) have been investigated for the first time.

\section{Materials and methods}

\section{Synthesis of CaP particles by FSP}

In the FSP system, metal precursor type, its chemical solubility, solvent type, decomposition temperature of metal precursors, as well as the flame operating parameters should all be considered to obtain particles with the desired properties. Precursor and the solvent type in this study were selected in light of these critical points and relevant previous studies. In the literature, calcium oxide or calcium hydroxide was used as calcium precursors, tributyl phosphate as phosphate precursor, and 2-ethylhexanoic acid as the solvent (Loher et al. 2005; Döbelin et al. 2010; Hild et al. 2011). We used the acetate form of Ca precursor, and propionic acid as the solvent, which worked well with the acetate form of $\mathrm{Ca}$ precursor and provided high enough combustion enthalpy to decompose the precursors, sustain the flame, and to produce nanoparticles in our experimental range of $\mathrm{Ca} / \mathrm{P}$ precursor ratio.

Metal precursors, which were dissolved in propionic acid solvent, were carried into the premixed burner by means of a liquid pump and a two-phase nozzle with a capillary insertion, which was located in the center of the burner. Precursor solution was then sprayed into the flame by an oxygen dispersion gas, and ignited by 25 premixed methane/oxygen flamelets 


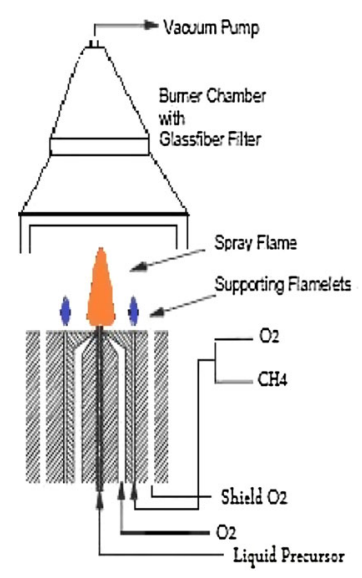

(a)

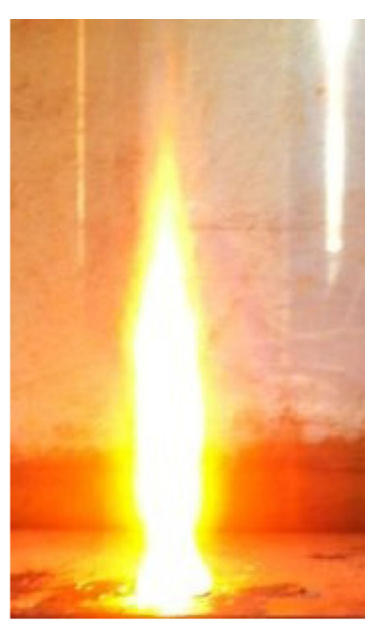

(b)
Fig. 1 a Schematic presentation of FSP system (Sel et al. 2014), b The burning spray of precursors

creating a stable main flame in which the particles were formed. Particles produced in the flame were collected on a vacuum applied glass fiber filter (GF/B) simultaneously. The burner and the chamber where the GF/B filter was placed were cooled to prevent overheating (Fig. 1).

Calcium phosphate was prepared using calcium acetate hydrate (99. $99 \%$ Sigma-Aldrich) dissolved in propionic acid (for synthesis $\geq 99 \%$, Merck) at $60{ }^{\circ} \mathrm{C}$ for $1 \mathrm{~h}$, and tributyl phosphate precursor in liquid form (assay $\geq 99 \%$, Sigma-Aldrich) was added after clear solution was observed ( $0.4 \mathrm{M}$ precursor solution). A range of different $\mathrm{Ca} / \mathrm{P}$ ratios from low to high (1.27, $1.38,1.61,1.95$, and 2.29) were studied. The liquid precursors were pumped through nozzle with the aid of a syringe pump (Watson, $323 \mathrm{~S} / \mathrm{D}$ ) at $7 \mathrm{rpm}(2.4 \mathrm{ml} / \mathrm{min}$ ), dispersed by oxygen $(9 \mathrm{l} / \mathrm{min})$ gas, and ignited with methane $(2 \mathrm{l} / \mathrm{min})$ and oxygen $(2 \mathrm{l} / \mathrm{min})$ premixed gases. The formed nanoparticles were collected on GF/B glass microfiber filter ( $1.0 \mu \mathrm{m}$, Whatman $)$ above the flame with the aid of a vacuum pump.

\section{Characterization of $\mathrm{CaP}$ particles}

X-ray diffraction (XRD), scanning electron microscopy (SEM), transmission electron microscopy-energy dispersive spectrometry (TEM-EDS), selected area electron diffraction (SAED), and high-resolution transmission electron microscopy (HRTEM) techniques were used for structural and morphological characterizations of the nanoparticles. XRD and SEM were used to determine the crystal phase and to investigate the general morphology. XRD analysis were performed by using Rigaku International Corporation Model:D/MAX2200/PC X-RAY Tube Cu:9407F8. Particles were analized using JEOL JSM 6335-F high resolution scanning electron microscope (HRSEM) at $15 \mathrm{kV}$. TEM and HRTEM were used for more detailed analyses - for atomic lattice imaging, nanoparticle size, crystal structure, and morphology of the particles. TEM was equipped with energy-dispersive $\mathrm{X}$-ray spectroscopy (EDS) to determine the surface elemental distribution. TEM investigations were performed on JEOL JEM 2100 HRTEM operating at $200 \mathrm{kV}$ (LaB6 filament) and equipped with an Oxford Instruments INCA X-Sight 6498 EDS system $\left(30 \mathrm{~mm}^{2}\right.$ ATW2 window, Oxford Inca Suite 4.05 software). Images were taken by Gatan Model 794 Slow Scan CCD Camera and also by Gatan Model 833 Orius SC200D CCD Camera. Elemental analyses for the precursors and the prepared nanoparticles were performed by inductively coupled plasma mass spectrometry (ICP-MS, Elmer DRC II model ICP-MS,). Brunauer-Emmett-Teller specific surface area $\left(S_{\mathrm{BET}}\right)$ measurements were used to determine the surface area of the particles produced using Micromeritics Gemini 2365 Automated Physisorption Analyzer. Samples were degassed at $250{ }^{\circ} \mathrm{C}$ for $2 \mathrm{~h}$ under $\mathrm{N}_{2}$ gas atmosphere prior to the analysis. The mean particle size was measured by HRTEM. To examine the crystallization $\mathrm{p}$ of the prepared nanoparticles, thermogravimetric analysis and differential thermal analysis (DTA) (Setaram Labsys) were performed. The temperature was programmed from room temperature to $900{ }^{\circ} \mathrm{C}$ at a heating rate of $10^{\circ} \mathrm{C} / \mathrm{min}$.

\section{Cell culture studies}

USC were isolated from spontaneously voided healthy human urine (male, 24 years) after obtaining the consent of Middle East Technical University Human Subjects Ethic Committee. The isolation procedure was performed as described in the literature (Zhang et al. 2008; Bharadwaj et al. 2011; Guan et al. 2014). The cells were cultivated in keratinocyte serum-free medium (KSFM; Invitrogen,USA) and embryonic fibroblast medium in a 1:1 ratio, $10 \%$ heat-inactivated fetal bovine serum, $1 \%$ Pen-Strep antibiotics, and supplemented with $5 \mathrm{ng} / \mathrm{ml}$ EGF and $50 \mathrm{ng} / \mathrm{ml}$ bovine pituitary extract. The cells were then passaged at 1:3 ratios. Immunophenotyping of 
stem cells was done by flow cytometric analysis. CD 73, CD105, and CD 44 were used as mesenchymal positive markers and CD 34 and CD 45 as negative markers. For bioactivity and cytotoxicity studies, nanoparticles were sterilized at $200{ }^{\circ} \mathrm{C}$ for $30 \mathrm{~min} .3 \times 10^{5}$ urine-derived cells were seeded per well in 24-well plates in osteogenic medium. Amorphous CaP nanoparticles in DMEM were added to each well at different final concentrations of particles of $5,10,25$, and $50 \mu \mathrm{g} / \mathrm{ml}$, respectively (Buschmann et al. 2012). Urine-derived stem cell culture in osteogenic medium without nanoparticles served as control. The cell viability assay was performed by PrestoBlue assay (Invitrogen, USA) at days 1, 3, 5, and 7 according to the manufacturer's guidelines. At days 7 and 14, ALP activities of the cells in all groups were determined. After 21 days of incubation, osteopontin expression was studied by FACS analysis. Sample size was $n=4$ for all conditions.

\section{Statistical analyses}

All data are presented as the mean \pm standard deviation (SD). One-way analysis of variance was used to determine statistically significant differences among groups, and pairwise comparisons were also performed with two-sample $t$ test with unequal variances. Statistical significance was considered for $p<0.05$. All statistical analyses were performed with SPSS Statistics 22.0

\section{Results}

\section{Characterization of nanoparticles}

The prepared nanoparticle powder collected on the GF/B filter was white in color and had specific surface

Table 1 Elemental analysis, specific surface area particles

\begin{tabular}{lll}
\hline $\begin{array}{l}\text { Ca/P molar ratio } \\
\text { (experimental) }\end{array}$ & $\begin{array}{l}\text { Ca/P ratio } \\
\text { (ICP results) }\end{array}$ & $\begin{array}{l}\text { Specific surface } \\
\mathrm{area}^{\mathrm{a}}\left(\mathrm{m}^{2} / \mathrm{g}\right)\end{array}$ \\
\hline 1.27 & 1.20 & $40.88 \pm 0.05$ \\
1.38 & 1.31 & $39.10 \pm 0.18$ \\
1.61 & 1.54 & $39.25 \pm 0.06$ \\
1.95 & 1.91 & $50.21 \pm 0.05$ \\
2.29 & 2.19 & $50.00 \pm 0.29$ \\
\hline
\end{tabular}

a Instrument's measurement error area (SSA) between 40 and $50 \mathrm{~m}^{2} / \mathrm{g}$, depending on the $\mathrm{Ca} / \mathrm{P}$ ratio (Table 1). The increasing $\mathrm{Ca} / \mathrm{P}$ ratio caused a slight increase in the SSA due to the increasing $\mathrm{Ca}$ concentration in the main flame environment, which in turn provided a larger number of seeds for particle formation, followed by their agglomeration and sintering before leaving the flame. Elemental analysis of particles after synthesis agreed well with the $\mathrm{Ca} / \mathrm{P}$ ratio in starting precursor solution (Table 1).

Scanning electron microscope was used for elemental microanalyses with energy-dispersive spectrometry (EDS) technique. For all samples, $\mathrm{Ca}, \mathrm{P}$, and $\mathrm{O}$ elements were detected. No contamination was observed.

All calcium phosphate particles that were studied in the experimental $\mathrm{Ca} / \mathrm{P}$ ratio range $(1.20,1.31,1.54$, 1.91, and 2.19) were of nanosizes. The representative low-magnification SEM and TEM images of a sample are given in Fig. 2, in which the polydisperse size distribution of particles can be seen. The particles had spherical shapes with loosely agglomerated structure.

In FSP process, particle formation takes place from gas-to-particle and/or droplet-to-particle mechanism. We occasionally observed these particles in the SEM images with small magnification, and they are shown in Fig. 3. Some particles formed from droplets are circled for visibility in the SEM images below. Inset in image (e) shows a close-up picture of one of the particles, while image (f) shows the crust of a particle.

Occasional large primary particle observations in our SEM and TEM pictures indicates that, the particle formation in our system follows both gas to particle and droplet to particle mechanism under the conditions we studied. Majority of the primary particle diameters were less than $100 \mathrm{~nm}$.

The average primary particle size was determined as $23 \mathrm{~nm}$ by counting more than 500 particles in TEM pictures, as shown in histogram in Fig. 4. Average particle diameter was almost the same for all samples, which was expected, because the flame reactor's operating conditions were kept the same in all the experiments. Smallest particle size was about $10 \mathrm{~nm}$.

TEM images of different calcium phosphate ratio samples are given in Fig. 5. The particles were amorphous, which indicates the fast cooling of the particles after their formation, and leaving the flame without crystallizing. Yet, fused primary particles with sintered necks were also observed. In Fig. 5f, HRTEM-SAED pattern for sample $\mathrm{Ca} / \mathrm{P}: 1.31$ is given, which shows the amorphous nature of the 


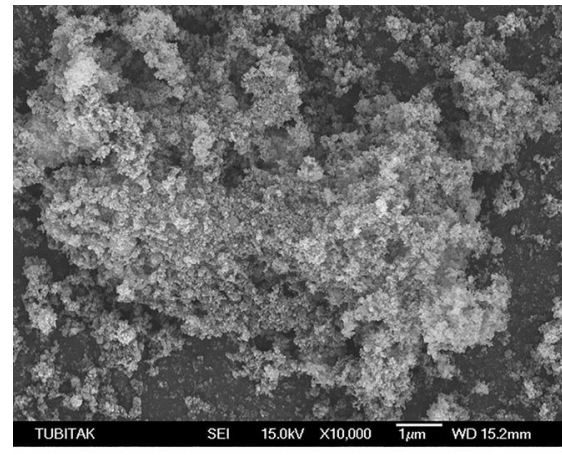

(a)

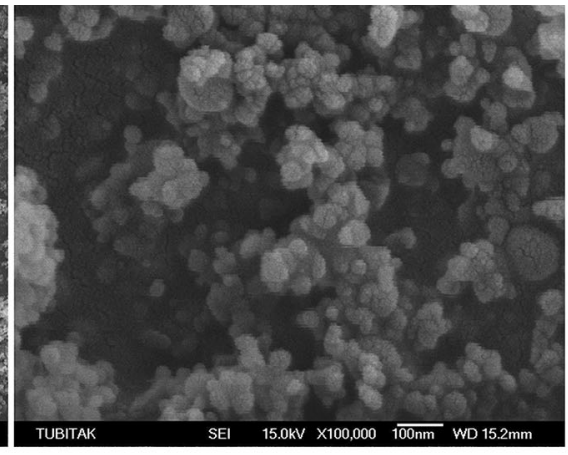

(b)

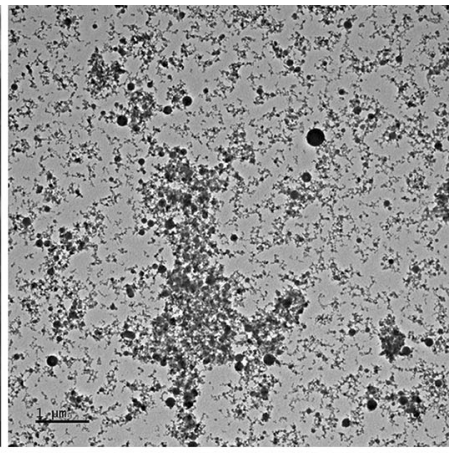

(c)

Fig. 2 Representative low-magnification SEM a (scale 1 micron) b (scale $100 \mathrm{~nm}$ ), and TEM c (scale 1 micron) images showing loose agglomerations $(\mathrm{Ca} / \mathrm{P}: 1.20)$

product. However, as the $\mathrm{Ca} / \mathrm{P}$ ratio was increased, crystallinity increased as confirmed by XRD results. Figure 6 shows a crystalline nanoparticle's HRTEM image and the corresponding Fast Fourier transform (FFT) diffractogram for sample $\mathrm{Ca} / \mathrm{P}: 2.19$, which had the highest $\mathrm{Ca} / \mathrm{P}$ ratio and crystallinity. The $\mathrm{d}$ spacing is measured as $0.28 \mathrm{~nm}$ which corresponds to $0.2814 \mathrm{~nm}$ of [2 $\left.\begin{array}{lll}2 & 1 & 1\end{array}\right]$ hydroxyapatite phase (JCPDS 09-0432; hexagonal, Space Group: P63/m (176); $\mathrm{a}=9.418 \mathrm{~A}^{\mathrm{o}}$ and $\mathrm{c}=6.884 \mathrm{~A}^{\circ}$ ). A sharp peak is observed at two theta $=31.77$ degree in XRD diagram (Fig. 7), which also corresponds to [2 111 l] hydroxyapatite phase.

The XRD pattern of the as-prepared calcium phosphate powders showed broad HA reflections especially up to the $\mathrm{Ca} / \mathrm{P}$ ratio of 1.54 , indicating the amorphous nature of the particles (Fig. 7). In all the powders prepared, sharp peaks corresponding to crystalline $\mathrm{CaO}$, and broad reflections of amorphous beta-CPP and alpha-TCP existed.

Thermal behavior of the particles up to the temperature of $900{ }^{\circ} \mathrm{C}$ by TG-DTA showed mass losses at $\sim 200, \sim 500,700{ }^{\circ} \mathrm{C}$, and at around $800{ }^{\circ} \mathrm{C}$ for $\mathrm{Ca} / \mathrm{P}$ ratios above 1.54 (Fig. 8). 3-4\% mass losses at all compositions were thought to be due to desorption of water in the samples

Isolation and characterization of urine-derived stem cells (USC)

The USC were isolated from healthy 24-year-old men donors and cultured in KSFM: EFM (1:1) medium. In 3-5 days of isolation, single cells were adhered to plate surface (Fig. 9a); then medium was changed three times a week. The remaining particles from urine were removed by medium change. Since cells were grown from single adherent cell, they form cobblestone like colonies (Fig. 9b). The confluency was reached at around 10-12 days after isolation (Fig. 9c). The cells were passaged at 1:3 ratio after 12 days of incubation.

The characterization of USCs was studied by analyzing surface marker expression of cells at passage 3 . The results were in correlation with those of the literature (Zhang et al. 2008; Bharadwaj et al. 2011). USCs were positive for C73, CD105, and CD44 and negative for CD45 and CD31 (Fig. S1).

In vitro cytotoxicity and bioactivity studies of calcium phosphate nanoparticles

PrestoBlue staining of living cells was performed to measure cellular viability of USC exposed to $\mathrm{CaP}$ nanoparticles at predetermined time intervals. Relative viabilities of all compositions were compared (Fig. 10). There was no statistically significant difference among different compositions. For all time periods, percent viabilities of all groups were around $85 \%$.

The bioactivity of flame-synthesized particles was studied by measuring ALP enzyme activity. To test whether the as-prepared particles have a positive effect on osteogenic differentiation, biochemical ALP assay was performed, and the results are given as specific enzyme activity ( $\mathrm{nmol} / \mathrm{min} / \mathrm{mg}$ protein). The effects of different concentrations $(5,10,25$ and $50 \mu \mathrm{g} / \mathrm{ml}$ ) of all compositions of calcium phosphate particles (1.20-2.19 $\mathrm{Ca} / \mathrm{P}$ ratio range) on th viability and the osteogenic differentiation of cells were studied (Fig. 11). Slight decreases in ALP activity of cells 

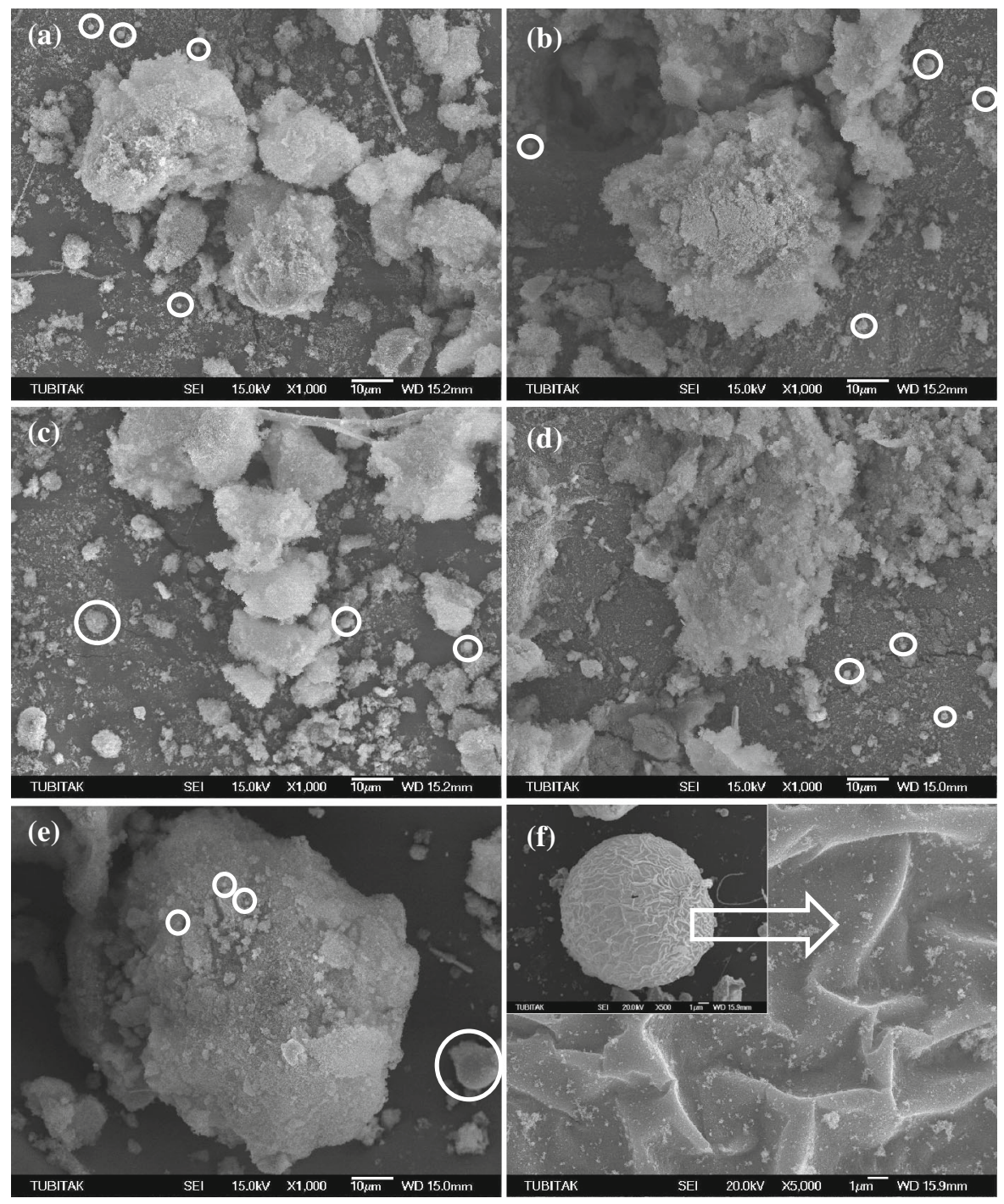

Fig. 3 SEM images of CaP samples, Ca/P: a 1.20, b 1.31, c 1.54, d 1.91, e 2.19, f close-up picture of a particle surface

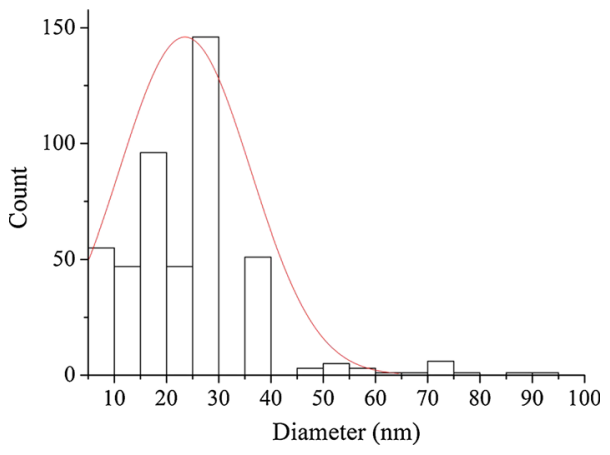

Fig. 4 Histogram of nanoparticle size treated with two highest $\mathrm{Ca}: \mathrm{P}$ ratios at $50 \mu \mathrm{g} / \mathrm{ml}$ concentration were observed at day 7 . There were no statistically significant differences among concentrations and compositions at two-time points. However, ALP activities of cells for all groups decreased at day 14 for each group.

\section{Discussion}

The advances in nanomaterial synthesis methods have enabled application of nanostructured materials to 

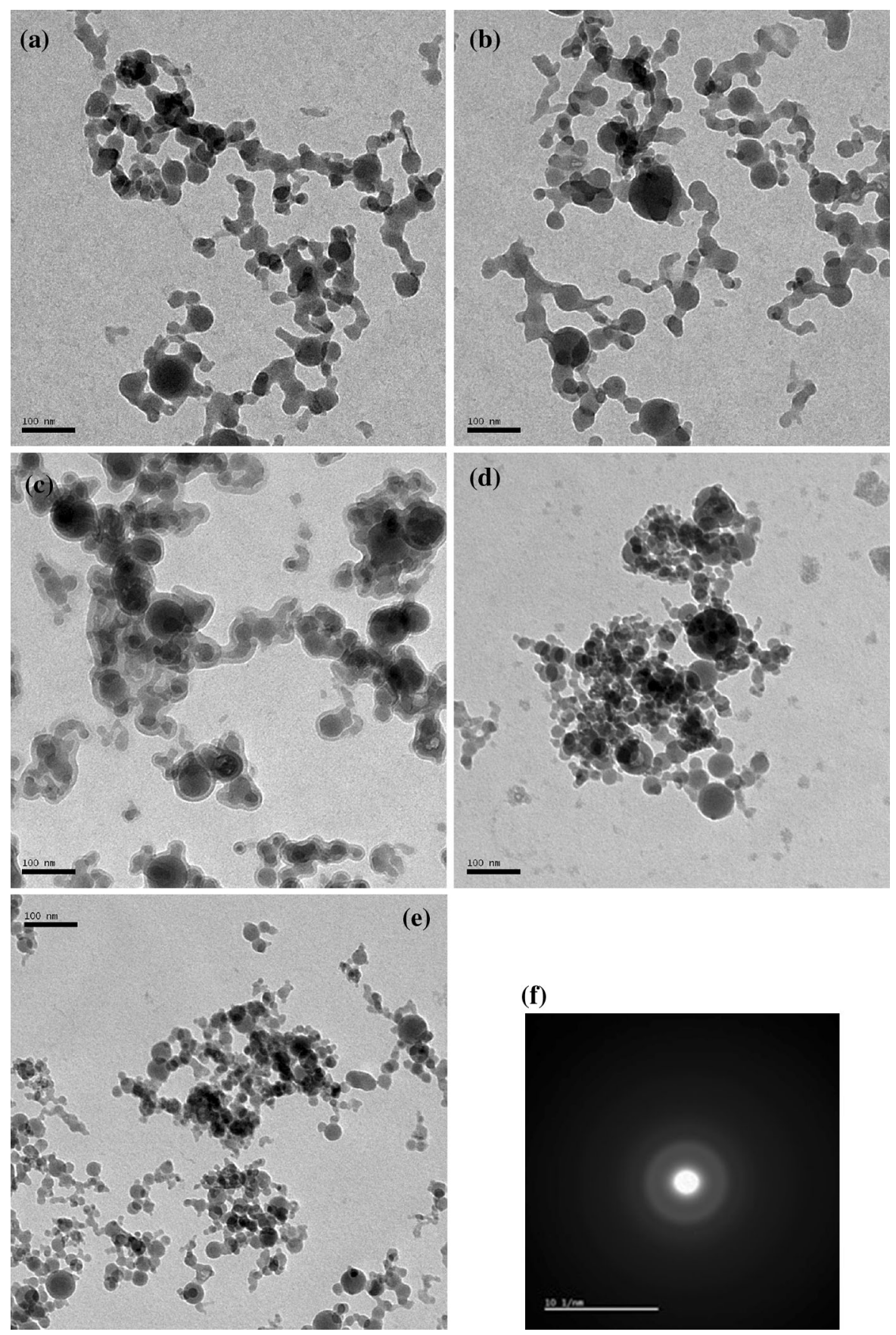

Fig. 5 TEM images of different calcium phosphate ratio samples a 1.20, b 1.31, c 1.54, d 1.91, e 2.19 at 100 nm scalebar, f HRTEMselected area electron diffraction (SAED) pattern for sample $\mathrm{Ca} / \mathrm{P}: 1.31$ that reveals amorphous phase

various fields. In medical treatments, nanostructured materials are preferred and used in safe dosage levels (not to exceed ppm levels). It has been reported that nanosized particles are preferred for engineered tissues, since smaller size and larger surface area result in an increase in protein adsorption, improved cell adhesion on materials, and gene expression induction (Brunner et al. 2006; 2007b; Stanley 2014).

Over the last decade, interest in nanoparticles produced by FSP to be applied in biomedical field 


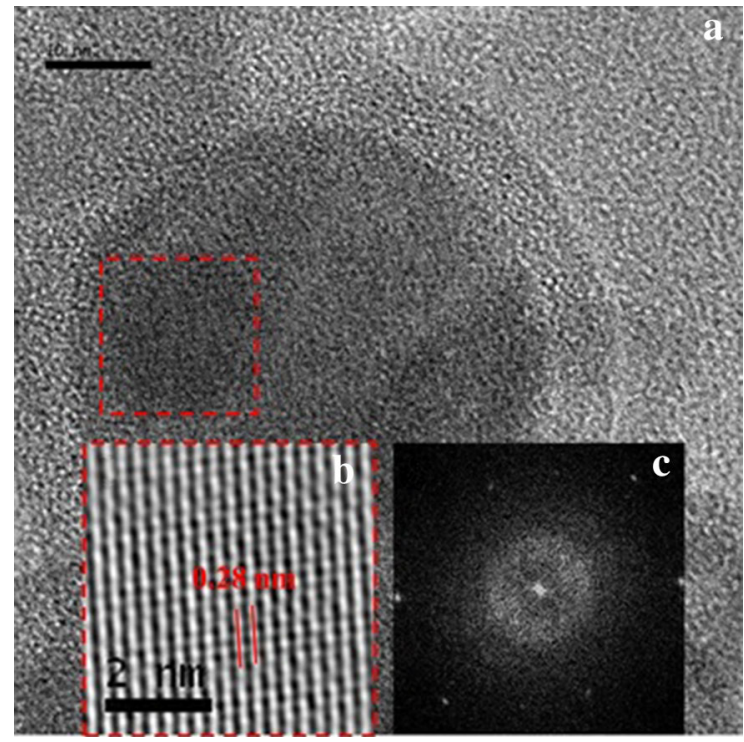

Fig. 6 a HRTEM image of sample Ca/P: 2.19 (scale: $10 \mathrm{~nm}$ ), b magnified and filtered view of the selected part in image a (scale: $2 \mathrm{~nm}$ ) and c corresponding FFT diffractogram

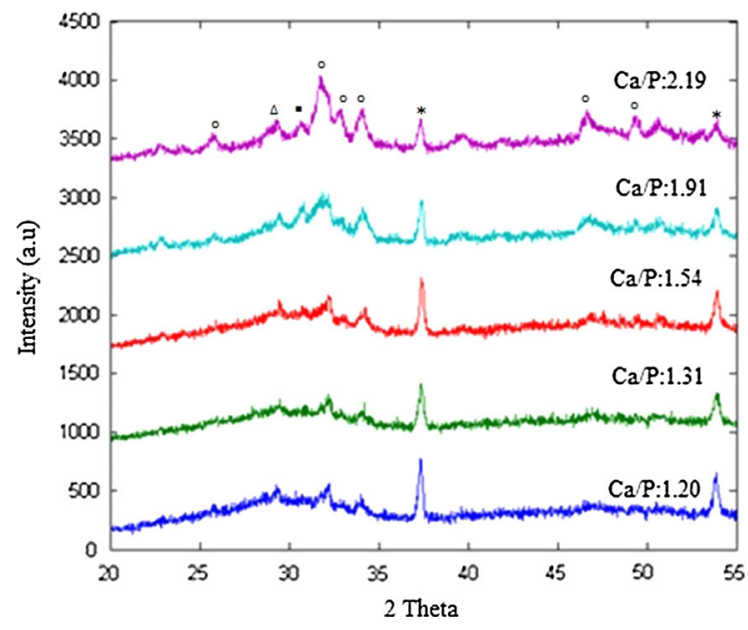

Fig. 7 XRD spectrum of the as-prepare d calcium phosphate particles (open circle) hydroxyapatite, (open triangle) beta$\mathrm{CPP}$, (filled square) alpha TCP, (asterisk) $\mathrm{CaO}$

has increased. In this method, formulation and the chemistry of precursors, and the choice of solvent have crucial influences on particle formation. Here, we used calcium acetate hydrate precursor and propionic acid solvent in our system to produce calcium phosphate particles. The prepared particles were of nanosize and in spherical shape, and showed a polydisperse size distribution, as observed in Fig. 2 and 3. SEM and

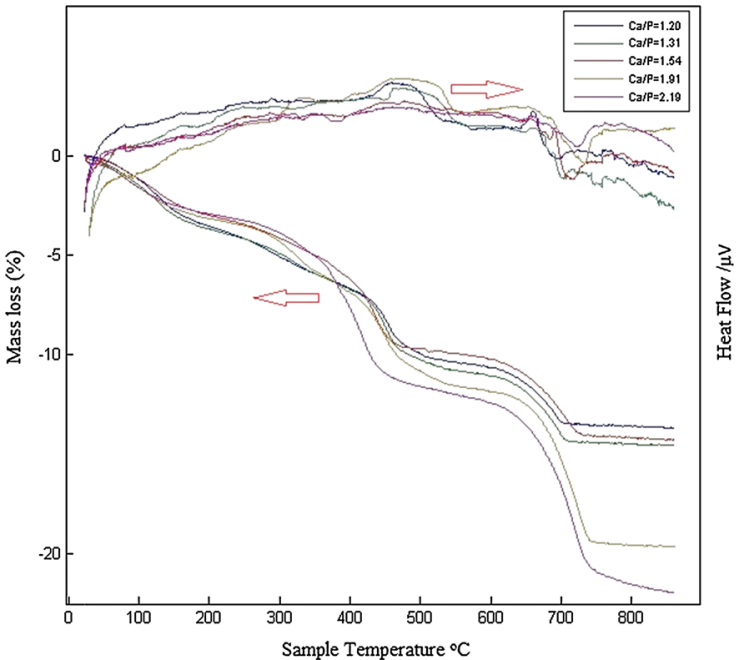

Fig. 8 TGA-DTA curves of flame synthesized calcium phosphate nanoparticles

TEM results indicated that the primary particle formation in our system follows both gas-to-particle and droplet-to-particle mechanisms under the conditions we studied. In the FSP process, particle-formation mechanism depends on several parameters. The most important of these parameters are flame temperature (decided by the heat of combustion of the solvent and premixed flame operating conditions), liquid precursor flow rate and concentration (effects the particle residence time and the degree of sintering), and dispersion oxygen's flow rate (effects the liquid droplets size in the spray) (Teoh et al. 2010; Camenzinda et al. 2010; Jossen et al. 2005; Sel et al. 2014). With high flame temperatures and enough residence time in the flame, the particle-formation mechanism follows gas-to-particle path only. Otherwise, dropletto-particle formation would also take place, because precursor liquid droplets do not completely evaporate within the flame. During the solvent evaporation, a concentration gradient can occur within the droplet causing a supersaturation of the precursors on the droplet surface forming a crust and even hollow eggshell particles, as shown in Fig. 3.

The SEM and TEM pictures show agglomeration of the primary particles as well, which is generally the case for the FSP-produced nanoparticle powders due to their need to minimize their surface free energy. TEM images indicated spherical primary particles with necking and forming chain-like aggregate structures. This kind of morphology is desired because 


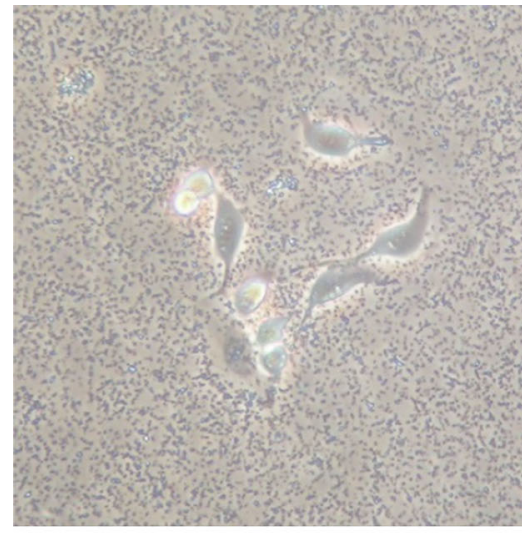

(a)

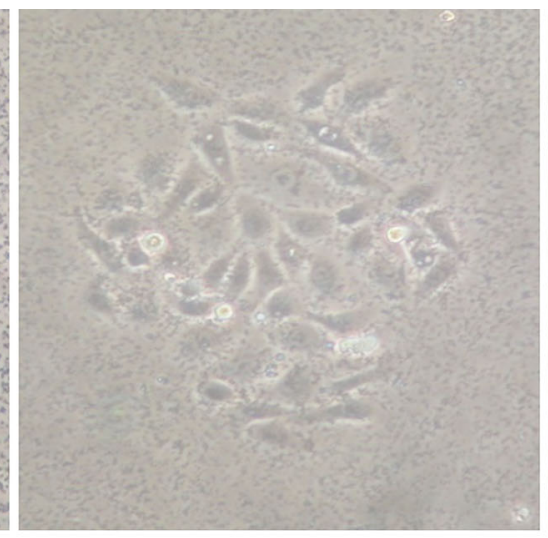

(b)

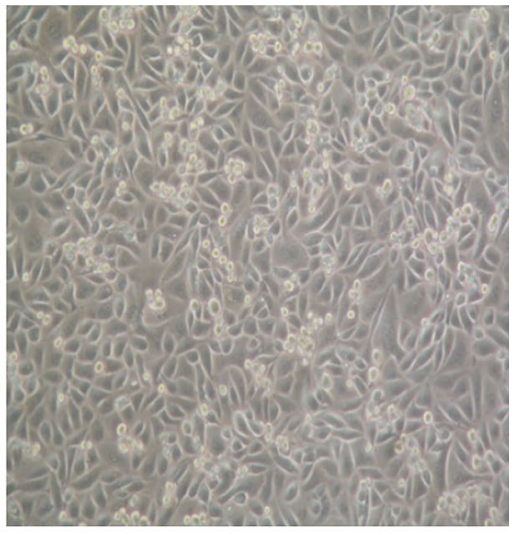

(c)

Fig. 9 Phase-contrast micrographs of urine-derived stem cells at a days $3-5$, b day 7 , $\mathbf{c}$ day 10

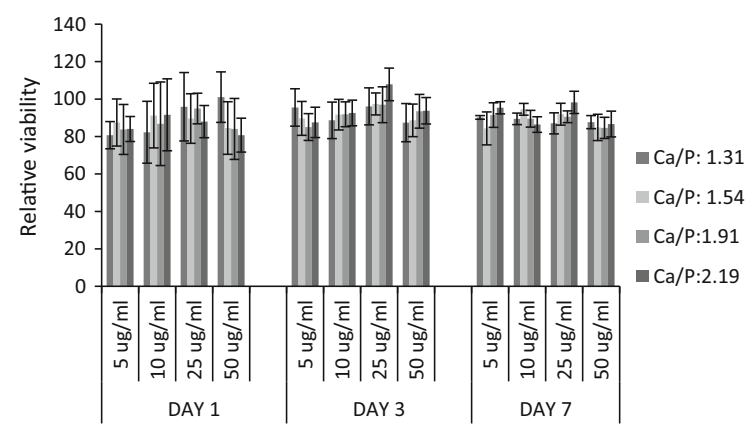

Fig. 10 Relative cell viability of USC exposed to different concentrations of calcium phosphate nanoparticles at different $\mathrm{Ca} / \mathrm{P}$ ratios. Cells that were not exposed to nanoparticles were used as control $(n=5)$

interconnective microporosity facilitates cell adhesion and body fluid circulation, and improves osteoconductive properties (Loher et al. 2005). When solvent + precursor mixture is sprayed into the flame environment, precursors pass through a series of reactions forming the particle nucleus. This is then followed by the particle growth through agglomeration and sintering, depending on the residence time in the flame (Teoh et al. 2010). The existence of particle necking indicates that some particles stayed long enough in the high-temperature regions of the flame to collide and stick together, and form chain-like aggregates.

HRTEM images of nanoparticles at low $\mathrm{Ca} / \mathrm{P}$ ratios showed no atomic lattice fringes, and corresponding FFT diffractograms in lower $\mathrm{Ca} / \mathrm{P}$ ratios were considered as an evidence for amorphous structures (Fig. 6).
However, relatively higher crystallinity was observed for the samples with $\mathrm{Ca} / \mathrm{P}$ ratio larger than 1.54 . This observation was also supported by XRD analysis.

Thermal behavior of synthesized calcium phosphate nanoparticles was analyzed by thermogravimetric (TG) and differential thermal analysis (DTA) as given in Fig. 8. All five calcium phosphate compositions $(1.20,1.31,1.54,1.91$, and 2.19) showed similar exothermic peaks and similar amounts of weight losses, but the last two samples had another phase change around $800{ }^{\circ} \mathrm{C}$. The weight loss up to $200{ }^{\circ} \mathrm{C}$ is an indicator of adsorbed water evaporation, and up to $\sim 400{ }^{\circ} \mathrm{C}$, it is thought to be due to the lattice water evaporation (Sun et al. 2010). Decomposition temperature and crystallization onset temperature in TG analysis differ with respect to synthesis methods of calcium phosphates. In their study, Loher et al. decided that the weight loss up to $600{ }^{\circ} \mathrm{C}$ was due to water vapor and $\mathrm{CO}_{2}$ loss from their sample (Loher et al. 2005). From 600 to $1250{ }^{\circ} \mathrm{C}$, it was interpreted as crystallization reaction and phase change. In our work, observation of phase change at about $800{ }^{\circ} \mathrm{C}$ for higher $\mathrm{Ca} / \mathrm{P}$ ratios in DTA results suggests the formation of beta-TCP, which is a more stable form of alpha-TCP (Cho et al. 2009). The onset of phase transition started at about $500{ }^{\circ} \mathrm{C}$ according to our TGA results. After $700{ }^{\circ} \mathrm{C}$, there was a plateau-like gradual decrease on TGA curves suggesting the crystallization of the particles.

Physical and chemical properties of calcium phosphate particles determine the biological activity. The changes in chemical composition as various calcium 


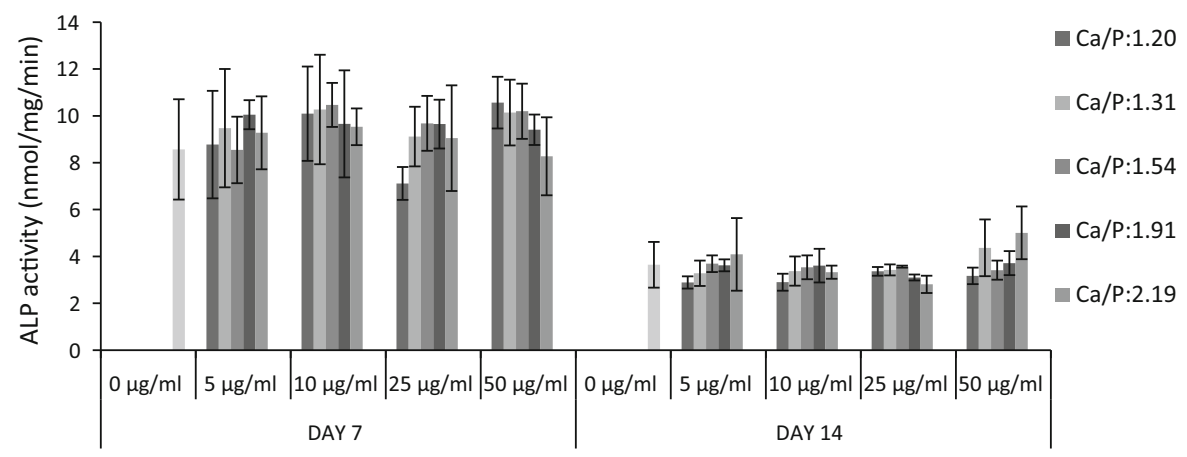

Fig. 11 ALP activity of USC that are exposed to different concentrations of $\mathrm{CaP}$ nanoparticles of different compositions in osteogenic medium. Cells unexposed to nanoparticles were used

to phosphate molar ratio and changes in structural properties such as crystallinity, porosity and particle size result in differences in behaviors of particles (Liu et al. 2008). In this study, properties of nanoparticles of calcium phosphates at different $\mathrm{Ca} / \mathrm{P}$ molar ratios were studied. Biological characterization of particles was conducted by cell-viability and ALP-activity studies. In previous studies, cytotoxic effect of $\mathrm{CaO}$ impurity was observed with a decrease in cell viability (Liu et al. 2008; Mohn et al. 2011). Nanoparticles with $\mathrm{CaO}$ impurity showed no cytotoxic effect on USC cells when added to the culture media at different concentrations in our study (Fig. 10). To examine the effect of amorphous calcium phosphate nanoparticles on osteogenic differentiation of the USC, ALP enzyme activity of cells was determined (Fig. 11). Particles were tested on monolayer cell culture. There was no statistically significant difference in each day between cells with different compositions and concentrations of nanoparticles, and cells without nano calcium phosphates. Between two time points, a numerical difference was found, which correlated with osteogenic differentiation progress (Lock and Liu 2011). Among all concentrations studied in the range of $1.20-2.19 \mathrm{Ca} / \mathrm{P}$ ratio, $50 \mu \mathrm{g} / \mathrm{ml}$ dose of calcium phosphate induction showed relatively higher enzyme activity level than that of control. Meanwhile, there was a slight decrease in the enzyme activity with the increasing calcium-to-phosphate ratio in $50 \mu \mathrm{g} / \mathrm{ml}$ dose groups. As stated above, the physical properties of nanoparticles influence the biological properties of material. Characterization results revealed that particles with the increasing calcium-to-phosphate ratio had more agglomeration, and showed more crystalline as control $(n=4)$. ALP activities of USC at day 7 were statistically higher than that observed at day 14 for all groups $(p<0.05)$

behaviors according to XRD patterns, HRTEM images, and FFT diffractograms. The increasing crystallinity decreases the dissolution rate of calcium phosphate particles (Chai et al. 2012). Therefore, it is not surprising to observe a relative decrease in the osteoinductive effect of calcium phosphates at higher ratios, which is in agreement with the literature (Yuan et al. 2010). These results allow us to claim that flamesynthesized amorphous calcium phosphate nanoparticles are cytocompatible and bioactive materials, and show their potential to integrate into 3D scaffolds or injectable bone cements.

\section{Conclusion}

In recent years, nanosized ACPs have received increased attention from the researchers for the development of bone biomimetic nanocomposites with different polymers for engineering of bone, due to their high solubility and surface reactivity in biological environment, capacity to improve mechanical properties, biomineralization, and bone regeneration in nanocomposite scaffolds. This study reveals that amorphous nanometer-sized calcium phosphate particles can be synthesized by the FSP method in time- and cost-effective manner in our proposed FSP system. The synthesized particles do not contain any unstable free ion or radical that could have resulted from pyrolysis, which can pose a risk for cytocompatibility. We have shown that the as-prepared nanosized calcium phosphates at different $\mathrm{Ca} / \mathrm{P}$ ratios at a $5-50 \mu \mathrm{g} / \mathrm{ml}$ range of doses were not toxic on USC and caused an improvement in the osteogenic 
differentiation of these cells. The results of the in vitro cell culture studies and characterization support the compatibility of the particles synthesizes for biomedical applications. For future studies, nano-sized ACPs will be used for the preparation of nanocomposite scaffolds mimicking the bone matrix biologically and biomechanically, using different scaffold processing techniques and the development of functional constructs using the easily obtained USC for the regeneration of bones.

Acknowledgments The authors would like to acknowledge with thanks the financial support from the METU BAP-07-022013-00, and The Scientific and Technological Research Council of Turkey (TUBITAK, Grant No 106M232). Sibel Ataol was supported financially by TUBITAK (Grant No 112T749).

Conflict of interest There is no conflict of interest for the study.

\section{References}

Alves Cardoso D, Jansen JA, Leeuwenburgh SCG (2012) Synthesis and application of nanostructured calcium phosphate ceramics for bone regeneration. J Biomed Mater Res B Appl Biomater 100:2316-2326

Barrère $\mathrm{F}$, van Blitterswijk $\mathrm{C}$, de Groot $\mathrm{K}$ (2006) Bone regeneration: molecular and cellular interactions with calcium phosphate ceramics. Int J Nanomed 1:317-332

Bharadwaj S, Liu G, Shi Y et al (2011) Characterization of urine-derived stem cells obtained from upper urinary tract for use in cell-based urological tissue engineering. Tissue Eng Part A 17:2123-2132

Blokhuis TJ, Arts JJC (2011) Bioactive and osteoinductive bone graft substitutes: definitions, facts and myths. Injury 42(Suppl 2):S26-S29

Boccaccini AR, Erol M, Stark WJ et al (2010) Polymer/bioactive glass nanocomposites for biomedical applications: a review. Compos Sci Technol 70:1764-1776

Brunner TJ, Wick P, Manser P et al (2006) In vitro cytotoxicity of oxide nanoparticles: comparison to asbestos, silica, and the effect of particle solubility. Environ Sci Technol 40:4374-4381

Brunner TJ, Bohner M, Dora C et al (2007a) Comparison of amorphous TCP nanoparticles to micron-sized $\alpha$-TCP as starting materials for calcium phosphate cements. J Biomed Mater Res Part B Appl Biomater 83:400-407

Brunner TJ, Grass RN, Bohner M, Stark WJ (2007b) Effect of particle size, crystal phase and crystallinity on the reactivity of tricalcium phosphate cements for bone reconstruction. J Mater Chem 17:4072

Buschmann J, Härter L, Gao S et al (2012) Tissue engineered bone grafts based on biomimetic nanocomposite PLGA/ amorphous calcium phosphate scaffold and human adipose-derived stem cells. Injury 43:1689-1697
Camenzinda A, Caserib WR, Pratsinis SE (2010) Flame-made nanoparticles for nanocomposites. Nano Today 5:48-65

Chai YC, Carlier A, Bolander J et al (2012) Current views on calcium phosphate osteogenicity and the translation into effective bone regeneration strategies. Acta Biomater 8:3876-3887

Chen F, Zhu Y, Wu J et al (2012) Nanostructured calcium phosphates: preparation and their application in biomedicine. Nano Biomed Eng 1:41-49

Cho JS, Kang YC (2008) Nano-sized hydroxyapatite powders prepared by flame spray pyrolysis. J Alloy Compd 464:282-287

Cho JS, Jung DS, Han JM, Kang YC (2009) Nano-sized $\alpha$ and $\beta$ TCP powders prepared by high temperature flame spray pyrolysis. Mater Sci Eng, C 29:1288-1292

Cho JS, Chung C-P, Rhee S-H (2010) Bioactivity and osteoconductivity of biphasic calcium phosphates. Bioceram Dev Appl 1:1-3

Combes C, Rey C (2010) Amorphous calcium phosphates: synthesis, properties and uses in biomaterials. Acta Biomater 6:3362-3378

Döbelin N, Brunner TJ, Stark WJ et al (2010) Thermal treatment of flame-synthesized amorphous tricalcium phosphate nanoparticles. J Am Ceram Soc 93:3455-3463

Dorozhkin SV (2010) Nanosized and nanocrystalline calcium orthophosphates. Acta Biomater 6:715-734

El Briak-Benabdeslam H, Ginebra MP, Vert M, Boudeville P (2008) Wet or dry mechanochemical synthesis of calcium phosphates Influence of the water content on DCPD-CaO reaction kinetics. Acta Biomater 4:378-386

Fathi MH, Hanifi A, Mortazavi V (2008) Preparation and bioactivity evaluation of bone-like hydroxyapatite nanopowder. J Mater Process Technol 202:536-542

Guan JJ, Niu X, Gong F, Guo SC (2014) Biological characteristics of human-urine-derived stem cells : potential for cell based therapy in neurology. Tissue Eng Part A 20:1-13

He W, Andersson M, de Souza PPC et al (2013) Osteogenesisinducing calcium phosphate nanoparticle precursors applied to titanium surfaces. Biomed Mater 8:035007

Hench LL, Polak JM (2002) Third-generation biomedical materials. Science 295:1014-1017

Hild N, Schneider OD, Mohn D et al (2011) Two-layer membranes of calcium phosphate/collagen/PLGA nanofibres: in vitro biomineralisation and osteogenic differentiation of human mesenchymal stem cells. Nanoscale 3:401-409

Hild N, Fuhrer R, Mohn D et al (2012) Nanocomposites of highdensity polyethylene with amorphous calcium phosphate: in vitro biomineralization and cytocompatibility of human mesenchymal stem cells. Biomed Mater 7:054103

Jillavenkatesa RA, Condreta SR (1998) Sol-gel processing of hydroxyapatite. J Mater Sci 3:4111-4119

Jossen R, Mueller R, Pratsinis SE, Watson M, Akhtar MK (2005) Morphology and composition of spray-flame-made yttria-stabilized zirconia nanoparticles. Nanotechnology 16:S609-S617

Liu H, Yazici H, Ergun C et al (2008) An in vitro evaluation of the $\mathrm{Ca} / \mathrm{P}$ ratio for the cytocompatibility of nano-to-micron particulate calcium phosphates for bone regeneration. Acta Biomater 4:1472-1479

Lobo SE, Livingston Arinzeh T (2010) Biphasic calcium phosphate ceramics for bone regeneration and tissue engineering applications. Materials (Basel) 3:815-826 
Lock J, Liu H (2011) Nanomaterials enhance osteogenic differentiation of human mesenchymal stem cells similar to a short peptide of BMP-7. Int J Nanomed 6:2769-2777

Loher S, Stark WJ, Maciejewski M et al (2005) Fluoro-apatite and calcium phosphate nanoparticles by flame synthesis. Chem Mater 1999:725-731

Loher S, Reboul V, Brunner TJ et al (2006) Improved degradation and bioactivity of amorphous aerosol derived tricalcium phosphate nanoparticles in poly(lactide-coglycolide). Nanotechnology 17:2054-2061

Maciejewski M, Brunner TJ, Loher SF et al (2008) Phase transitions in amorphous calcium phosphates with different $\mathrm{Ca} / \mathrm{P}$ ratios. Thermochim Acta 468:75-80

Madler L, Kammler HK, Mueller R, Pratsinis SE (2002) Controlled synthesis of nanostructured particles by flame spray pyrolysis. J Aerosol Sci 33:369-389

Mohn D, Doebelin N, Tadier S et al (2011) Reactivity of calcium phosphate nanoparticles prepared by flame spray synthesis as precursors for calcium phosphate cements. J Mater Chem 21:13963

Nagano M, Nakamura T, Kokubo T et al (1996) Differences of bone bonding ability and degradation behaviour in vivo between amorphous calcium phosphate and highly crystalline hydroxyapatite coating. Biomaterials 17:1771-1777

Overgaard S, Bromose U, Lind M et al (1999) The influence of crystallinity of the hydroxyapatite coating on the fixation of implants. Mechanical and histomorphometric results. J Bone Joint Surg Br 81:725-731

Pratsinis SE (1998) Flame aerosol synthesis of ceramic powders. Prog Energy Combust Sci 24:197-219

Sadat-Shojai M, Khorasani M-T, Dinpanah-Khoshdargi E, Jamshidi A (2013) Synthesis methods for nanosized hydroxyapatite with diverse structures. Acta Biomater 9:7591-7621

Salinas AJ, Esbrit P, Vallet-Regí M (2013) A tissue engineering approach based on the use of bioceramics for bone repair. Biomater Sci 1:40
Sarkar MR, Wachter N, Patka P, Kinzl L (2001) First histological observations on the incorporation of a novel calcium phosphate bone substitute material in human cancellous bone. J Biomed Mater Res 58:329-334

Sel S, Duygulu O, Kadiroglu U, Machin NE (2014) Synthesis and characterization of nano-V2O5 by flame spray pyrolysis, and its cathodic performance in Li-ion rechargeable batteries. Appl Surf Sci 318:150-156

Somrani S, Rey C, Jemal M (2003) Thermal evolution of amorphous tricalcium phosphate. J Mater Chem 13:888-892

Stanley S (2014) Biological nanoparticles and their influence on organisms. Curr Opin Biotechnol 28:69-74

Stark WJ (2011) Nanoparticles in biological systems. Angew Chem Int Ed Engl 50:1242-1258

Sun L, Chow L, Frukhtbeyn SA, Bonevich J (2010) Preparation and properties of nanoparticles of calcium phosphates with various Ca/P ratios. J Res Natl Inst Stand Technol 115:243-255

Teoh WY, Amal R, Mädler L (2010) Flame spray pyrolysis: an enabling technology for nanoparticles design and fabrication. Nanoscale 2:1324-1347

Tofighi A, Palazzolo R (2005) Calcium phosphate bone cement preparation using mechano-chemical process. Key Eng Mater 284-286:101-104

Yuan H, Fernandes H, Habibovic P et al (2010) Osteoinductive ceramics as a synthetic alternative to autologous bone grafting. Proc Natl Acad Sci U S A 107:13614-13619

Zhang Y, McNeill E, Tian H et al (2008) Urine derived cells are a potential source for urological tissue reconstruction. J Urol 180:2226-2233

Zhou H, Lee J (2011) Nanoscale hydroxyapatite particles for bone tissue engineering. Acta Biomater 7:2769-2781 